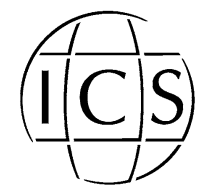

www.ics-elsevier.com

\title{
Image based methods to generate subject-specific musculoskeletal models for gait analysis
}

\author{
Lennart Scheys ${ }^{\mathrm{a}, \mathrm{b}, *}$, Ilse Jonkers ${ }^{\mathrm{b}}$, Filip Schutyser ${ }^{\mathrm{a}}$, Steven Pans ${ }^{\mathrm{c}}$, \\ Arthur Spaepen ${ }^{\mathrm{b}}$, Paul Suetens ${ }^{\mathrm{a}}$ \\ ${ }^{a}$ Medical Image Computing (Radiology-ESAT/PSI), University Hospital Gasthuisberg, Herestraat 4, \\ B-3000 Leuven, Belgium \\ ${ }^{\mathrm{b}}$ Department of Kinesiology, FABER/K.U.Leuven, Tervuursevest 101, B-3000 Leuven, Belgium \\ ${ }^{\mathrm{c}}$ Department of Radiology, University Hospitals Leuven, Herestraat 4, B-3000 Leuven, Belgium
}

\begin{abstract}
This paper describes a workflow, designed to build detailed, subject-specific musculoskeletal models using magnetic resonance (MR) images. Integration of semi-automated methods for segmentation of the bone reduced considerably the processing time. Furthermore, enhanced model quality was achieved compared to pure manual segmentation techniques. Two different semi-automated segmentation methods were tested and compared. The workflow was successfully applied to MR images of the lower extremity of a non-pathological adult subject: the femur, tibia and fibula were segmented along with lines of action of 25 muscles. (C) 2005 CARS \& Elsevier B.V. All rights reserved.
\end{abstract}

Keywords: Musculoskeletal modelling; Gait analysis; MR imaging; Segmentation

\section{Introduction}

Gait analysis has proven to be a valuable tool to assist clinical decision-making in patients with gait abnormalities. Especially in case of cerebral palsy (CP) its added value has been documented. Advanced biomechanical analysis of the muscle function during walking requires the use of musculoskeletal models describing the musculoskeletal geometry and force generating capacity of the muscles.

Today, most of the software packages for gait analysis rely on a generic model of the lower extremity, i.e. the musculoskeletal geometry of an average-sized adult male [1-6].

\footnotetext{
* Corresponding author. Medical Image Computing (Radiology-ESAT/PSI), University Hospital Gasthuisberg, Herestraat 4, B-3000 Leuven, Belgium.

E-mail address: lennart.scheys@uz.kuleuven.ac.be (L. Scheys).
} 
However in a paediatric population and especially in pathological conditions where bony deformations are present, such a generic model needs to be accommodated for differences in size and aberrant musculoskeletal geometry [7-10]. Two solutions are possible. One option is rescaling and deforming the generic model to approximate the patient's musculoskeletal geometry [11]. Alternatively, subject-specific models can be built based on medical images.

Previous research [12-14] has proven the potential to build accurate personalized models using magnetic resonance (MR) images. However, those methods are labor intensive and time consuming [11] since they rely on manual segmentation of the various muscles and bone structures of interest in the medical images. In this paper a workflow is presented for the definition of patient-specific musculoskeletal models integrating semiautomatic segmentation. This approach therefore requires less user input, and is therefore less time consuming than manual segmentation, while delivering models with a higher level of patient-specific detail than achieved by deformed generic models.

\section{Materials and methods}

A software tool for building subject-specific musculoskeletal models using MR images has been designed: Fig. 1. The output files of the defined musculoskeletal models have been formatted for use in SIMM (Software for Interactive Musculoskeletal Models) [15].

The model consists of three major parts: (1) body segments, containing one or more bones defined in a certain body coordinate system; (2) muscles, presented as a line model between origin and insertion as well as additional wrapping points when penetration of the muscle into the bones needs to be prevented, and finally (3) the positions of the different joints, interconnecting the different segments in the model space.

The following methods were integrated for definition/parameterization of each of these structures:

(1) A first method for segmentation of the bone is based on thresholding in each image slice. Based on a user-defined threshold, an iso-value contour is highlighted in the image. In case several contours are detected, the contour of interest can be selected by the user. This process is thereafter repeated for every slice of the medical image. If necessary, the
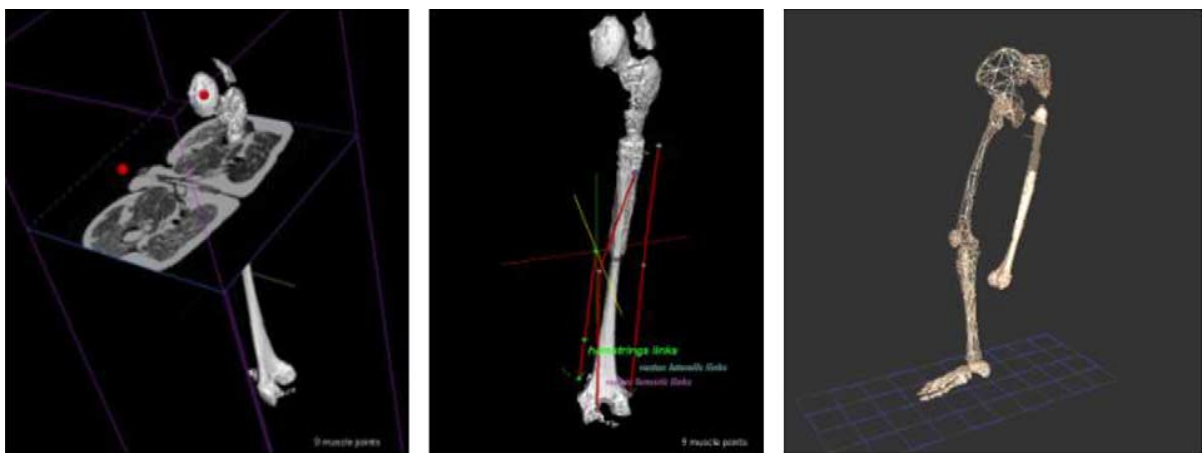

Fig. 1. A software tool has been developed to extract bone and muscle information from T1-weighted images (left and middle figure) suitable for SIMM (right figure). 
threshold value can be interactively adjusted during this process. Based on the resulting stack of successive contours in the 3D-space a surface is derived using interpolation.

A second, more automated method uses a 3D region-growing algorithm. It is based on the level set theorem by Osher and Sethian [16]. This theorem defines the segmentation problem as a three-dimensional surface propagating in time in the direction of its normals. The surface's evolution is determined by a speed function that, based on carefully selected local image properties and user defined parameters, chooses a proper distance and direction (inwards or outwards) for the local deformation of the evolving surface.

(2) The definition of a muscle as a line model requires detection of origin and insertion as well as intermediate wrapping points. The appropriate points are manually identified in successive image slices. In case the muscle path deviates from a straight line between origin and insertion, which can easily be checked by following the line model of the muscle while scrolling through the image slices, additional wrapping points can be added to the model. The software also allows the calculation of individual muscle lengths. Individual muscle points can thereafter be assigned to the relevant segments.

(3) The positions of the different joints serve as the points of application of the moments generated by the force-developing muscles and ligaments. These positions are closely related to the anatomical shape of the bones that define the joint under consideration so that inter-joint space is calculated based on the lengths of these bones. Additional user interaction is allowed for repositioning of the suggested joint axes, if necessary.

Finally, the definition of the musculoskeletal model is automatically transcribed in order to allow it to be directly imported in SIMM where the necessary biomechanical parameters can be studied. VTK [17] with Tcl/Tk [18] are applied for implementing this processing pipeline and packing it in a user-friendly GUI.

\section{Results}

With the software tool, a highly detailed personalized musculoskeletal model of a nonpathological adult subject ( 25 year) is created: Fig. 2.

MR-images were acquired using a three plane, T1-weighted spin-echo sequence of the subject lying supine. The field of view was the entire region of the lower extremity. To

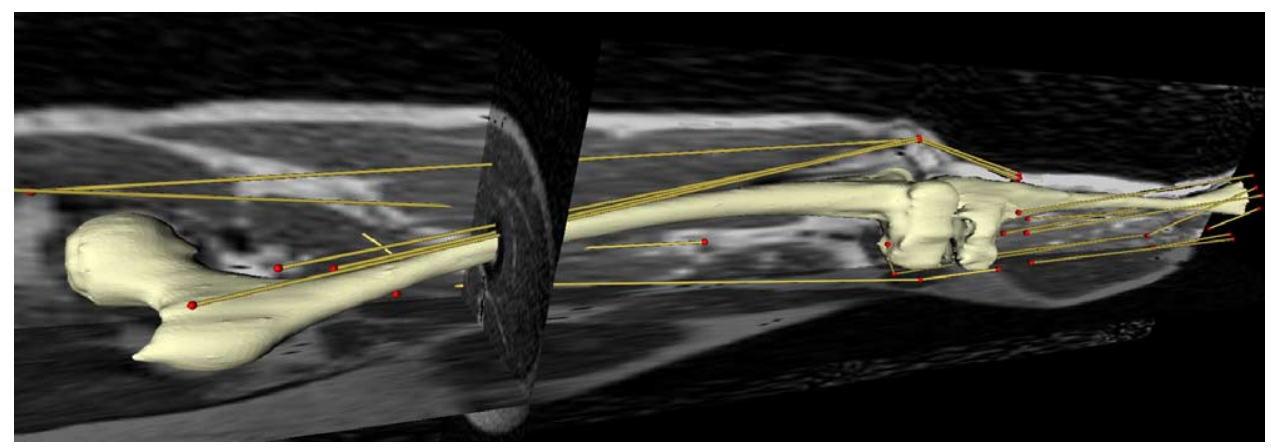

Fig. 2. The newly created right leg musculoskeletal model is co-visualized with a coronal, sagittal and axial slice from the MR image volume it was segmented from. 
speed up the process the slice thickness was not kept constant throughout the entire image volume. Along the shaft of the femur and the tibia a bigger slice thickness was used (12.58 $\mathrm{mm})$ than in the area of the knee, hip and ankle joint $(3 \mathrm{~mm})$ where more detail is needed to capture the articular surfaces and to define the kinematics [12]. The entire acquisition took about $11 \mathrm{~min}$.

Based on the acquired images, a personalized musculoskeletal model was defined. The structures from the tibia, the fibula and the femur were extracted twice, using each of the previously mentioned methods. However, to obtain a successful segmentation, a preprocessing step was necessary to remove the bias field that was present in the medical image. Before removal this was estimated using an iterative expectation-maximization strategy [19]. The resulting subject-specific bone surfaces have a resolution about 200 times as large as those in the original generic model $\left( \pm 20\right.$ points $/ \mathrm{cm}^{2}$ instead of the original \pm 0.1 points $/ \mathrm{cm}^{2}$ ). Consequently, the patient's skeletal structure is described with a much higher level of detail. In a final step the bone volumes are smoothed. Laplacian smoothing is used to adjust the point coordinates. This results in a polygonal mesh of the bone based on a more evenly distributed point cloud.

Next to these bone surfaces, lines of action of 25 major muscles of the lower extremity were defined. They included rectus femoris, vastus lateralis, vastus intermedius, vastus medialis, biceps femoris, semitendinosus, semimembranosus, adductor longus, adductor brevis, adductor magnus-inferior, adductor magnus-middle, adductor magnus-superior, gracilis, sartorius for the thigh area, and gastrocnemicus-lateral, gastrocnemicus-medial, soleus, extensor digitorum longus, extensor hallucis longus, tibialis posterior, tibialis anterior, flexor digitorum longus, flexor hallucis longus, peroneus longus, peroneus tertius for the shank area.

The attachment points of the muscle to the bone were identified according to the definition in Koshinen's study by scrolling through the image slices and picking an appropriate point in the last slice where the muscle is visible [20]. For tensor fascia lata and peroneus brevis, the insertions could not be identified unambiguously.

The defined lines of action contained for most muscles (rectus femoris; vastus lateralis, intermedius and medialis; sartorius; gracilis; semimembranosus; biceps femoris; gastrocnemius lateralis and medialis) at least one or two via points. Most of the via points were added at the level of the knee joint area in order to avoid that lines of action penetrate the femoral epicondyl or the tibial plateau. According to the method Arnold used, via points were introduced in order to accommodate the shape of the bones and joints. All the points were referenced to anatomy books [21-23].

\section{Discussion}

The developed software allows the definition of a personalized musculoskeletal model based on 2D MR cross-sectional images.

It should be noted that in the whole process, user experience is essential in the segmentation of the bone contours and even more in the digitization of the muscle points.

Despite the goal to reduce the time required for segmentation by integrating semiautomatic techniques, the test revealed that, the entire process is still very time-consuming because semi-automatic segmentation of the muscles failed so far. The current manual digitization of the muscles requires a substantial amount of time. 


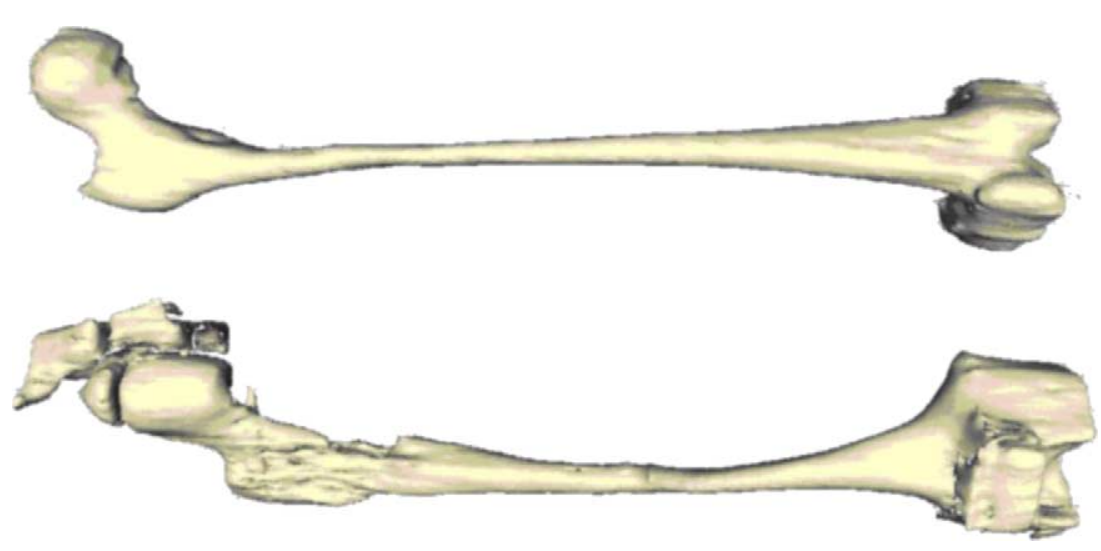

Fig. 3. Same femur segmented using the region growing algorithm (above) and the thresholding method (below). As can be seen, using the more automated region growing method instead of thresholding has a substantial positive effect on the quality of the segmentation.

Segmentation of the bone relied on the inside contour of cortical bone, which was easier to extract than the "outline" of cortical bone. Using thresholding, the outline of the cortical bone could only be captured in 1/10 of all 176 image slices.

The cortical layer is rather thin in the area of the joints so that the choice between using the inner or outer contour has little effect in those important regions. Along the shaft of the femur and the tibia, however, the cortical layer becomes substantial, resulting in a narrowed shaft.

Comparing both methods, region growing outperforms thresholding. Using region growing delivered a 50 percent time gain: segmenting a complete femur only takes 15 min, compared to $30 \mathrm{~min}$ when thresholding is used. Furthermore, the segmentations are of better quality, as can be seen in Fig. 3, containing less non-bone structures, especially in joint areas, where bone marrow comes into close contact with fatty tissue so that thresholding fails in distinguishing between the bone and the fatty tissue.

Musculoskeletal modelling has the potential to provide an accurate and efficient means of estimating muscle-tendon lengths and moment arms in vivo [12]. Incorporating MR images is tempting since it allows patient specific tailoring of the biomechanical analysis. However, the advancement of medical imaging methodologies allowing (semi-) automatic segmentation of bone and muscle structure seems crucial in providing the clinician with an efficient and user-friendly tool.

\section{Acknowledgements}

This article is dedicated to the memory of Johan Van Cleynenbreugel. His scientific enthusiasm and expertise, original ideas and support on every aspect of scientific research were an irreplacable contribution to this work.

We acknowledge the support from Flanders' Fund for Scientific Research (FWOVlaanderen) for this project on personalised musculoskeletal modelling of the pathological lower extremity using MR images: usability for biomechanical analysis (G.0570.05). 


\section{References}

[1] R.A. Brand, et al., A model of lower extremity muscular anatomy, Journal of Biomechanical Engineering 104 (1982) 304-310.

[2] T.M. Kepple, et al., A three-dimensional musculoskeletal database for the lower extremities, Journal of Biomechanics 31 (1998) 77-80.

[3] J.A. Friedreirch, R.A. Brand, Muscle architecture in the human lower limb, Journal of biomechanics 23 (1) (1990) $91-95$.

[4] G.N. Duda, et al., Variability of femoral muscle attachments, Journal of biomechanics 29 (9) (1996) $1185-1190$

[5] S.Y. White, H.J. Yack, D.A. Winter, A three-dimensional musculoskeletal model for gait analysis, Journal of Biomechanics 22 (8-9) (1989) 885-893.

[6] H.S. Kang, et al., The visible man: three dimensional interactive musculoskeletal anatomic atlas of the lower extremity, Radiographics 20 (2000) 286-297.

[7] E.B. Bleck, Orthopaedic Management in Cerebral Palsy, Mac Keith press, London, 1987, pp. $142-212$.

[8] J.R. Gage, Gait Analysis in Cerebral Palsy, Mac Keith press, London, 1991, p. 131.

[9] R.K. Belas, Developmental changes in the femur and the acetabulum in spastic paraplegia and diplegia, Developmental Medicine \& Child Neurology 11 (1969) 303-313.

[10] L. Fabeck, et al., Theoretic study of the decrease in the femoral neck anteversion during growth, Cells Tissues Organs 171 (2002) 269-275.

[11] A.S. Arnold, S.S. Blemker, S.L. Delp, Evaluation of a deformable musculoskeletal model: application to planning muscle-tendon surgeries for crouch gait, Annals of Biomedical Engineering (29) (2001) $1-11$.

[12] A.S. Arnold, et al., Accuracy of muscle moment arms estimated from MRI-based musculoskeletal models of the lower extremity, Computer Aided Surgery 5 (2000) 108-119.

[13] Z.A. Cohen, Computer-aided planning of patellofemoral joint OA surgery: developing physical models from patient MRI, Lecture Notes in Computer Science, Proceedings from the First Annual Conference on Medical Image Computing and Computer-Assisted Interventions, vol. 1496, 1496, pp. 9-20.

[14] D.K. Smith, et al., Validation of three-dimensional reconstructions of knee anatomy: CT vs MR imaging, Journal of Computer Assisted Tomography 13 (1989) 294-301.

[15] MusculoGraphics Inc a division of Motion Analysis Corporation. Simm. http://www.musculographics.com/ products/simm.html, 2003.

[16] J.A. Sethian, Level Set Methods and Fast Marching Methods, second edition, Cambridge University Press, 1999.

[17] Tcl Developer Xchange http://www.tcl.tk/.

[18] VTK - The visualisation toolkit http://www.vtk.org.

[19] K. Van Leemput, et al., Automated model-based bias field correction of $\mathrm{mr}$ images of the brain, IEEE Transactions on Medical Imaging 18 (10) (1999 October) 885-896.

[20] S.K. Koskinen, U.M. Kujala, Patellofemoral relationships and distal insertion of the vastus medialis muscle: a magnetic resonance imaging study in nonsymptomatic subjects and patients with patellar dislocation, Arthroscopy 8 (4) (1992) 465-468.

[21] D.R. Cahill, M.J. Orland, Atlas of Cross-Sectional Anatomy, Lea and Febiger, Philadelphia, 1984.

[22] R.C. Crafts, A Textbook of Human Anatomy, 3rd ed., 1985.

[23] R.S. Ledley, H.K. Huang, J.C. Mazzioti, Cross-sectional Anatomy: An Atlas for Computerized Tomography, Williams and Wilkens, Co., Baltimore, MD, 1977. 